\title{
PCR and ELISA vis-à-vis Microscopy for Detection of Bovine Anaplasmosis: A Study on Associated Risk of an Upcoming Problem in North India
}

\author{
Amrita Sharma, L. D. Singla, Paramjit Kaur, and M. S. Bal \\ Department of Veterinary Parasitology, College of Veterinary Sciences, Guru Angad Dev Veterinary and Animal Sciences University, \\ Ludhiana, Punjab 141004, India
}

Correspondence should be addressed to Amrita Sharma; amrita14887@gmail.com

Received 10 July 2014; Revised 1 February 2015; Accepted 1 February 2015

Academic Editor: Yudong Cai

Copyright (C) 2015 Amrita Sharma et al. This is an open access article distributed under the Creative Commons Attribution License, which permits unrestricted use, distribution, and reproduction in any medium, provided the original work is properly cited.

\begin{abstract}
This investigation demonstrates the status of bovine anaplasmosis caused by A. marginale in bovines from Submountain and Undulating Zone of Punjab. Out of 184 suspected animals, 25 (19.51\%), 47 (31.71\%), and 78 (68.75\%) were positive by microscopy, indirect ELISA, and PCR assay, respectively. The microscopy showed $29 \%$ sensitivity and $99 \%$ specificity, while ELISA showed $32 \%$ sensitivity and $79 \%$ specificity in concordance with PCR assay. Five false negative samples by mspl $\beta$ PCR were reconfirmed for Anaplasma spp. targeting 16S rRNA gene. The sequence analysis showed the presence for $A$. marginale specific restriction site, indicating variation in the local strains of the organism resulting in no amplification with msp1 $\beta$ gene primers. Of 82 samples positive by PCR, 57 were negative by ELISA indicating lower efficacy of ELISA to detect early anaplasmosis. The assessment of risk factor with results of PCR technique indicated that cattle (Odds ratio $=2.884)$, particularly those of age $>1$ years $($ Odds ratio $=$ 2.204) of district Pathankot (Odds ratio $=3.182$ ) of Submountain Zone (Odds ratio $=2.086$ ), were at high risk of anaplasmosis. All three districts of Submountain Zone are at higher risk indicating the impact of biotic and abiotic factors on the incidence of disease.
\end{abstract}

\section{Introduction}

Anaplasma marginale (Order Rickettsiales, Family Anaplasmataceae) causes pathogenic bovine anaplasmosis [1]. The organism multiplies within the erythrocytes of the host, resulting in extravascular haemolysis and acute anaemia, morbidity, and mortality in some cases [2]. Bovine anaplasmosis occurs in tropical and subtropical areas throughout the world and the disease is a major constraint to cattle production in many countries. Punjab state located in latitude $29^{\prime \prime} 30^{\prime} \mathrm{N}-32^{\prime \prime} 32^{\prime} \mathrm{N}$ and longitude $73^{\prime \prime} 55^{\prime} \mathrm{E}-76^{\prime \prime} 50^{\prime} \mathrm{E}$ provides favourable conditions for flare-up of the disease. In North India, 20 outbreaks of bovine anaplasmosis occurred during the period of January to June 2013, out of which 5 were reported from Jammu and Kashmir, 6 from West Bengal, and 9 (45\%) from Punjab, indicating the threats posed on livestock by the disease (http://www.oie.int/wahis_2/public/ wahid.php/Diseaseinformation/statusdetail). Thus an estimated more than 300 million of bovine population is at risk in Punjab (Livestock Census 2007). A wide variety of biological and mechanical agents are responsible for the transmission of this infection, amongst which Boophilus microplus is the most important vector in Punjab. After an acute phase of infection, animals may remain chronically infected carriers for years [3]. The level of parasitemia in carriers is below the threshold of detection by microscopy which has the detection limit of about 0.03 percent. The overall sensitivity of this method is $10^{6}$ infected erythrocytes per $\mathrm{mL}$ of blood. Moreover, it is time consuming and there is a need of an experienced eye to differentiate the pathogen from the related organisms including artefacts. Thus this this method is not recommended for the characterization of persistently infected cattle. Subinoculation of A. marginale infected erythrocytes into susceptible, splenectomized calves has been considered as the "gold standard" for detection of latent infection in cattle, but it is not practical for routine testing. Serological tests, even though developed, lack the required specificity and sensitivity for a reliable 
diagnosis. However, these tests for antibody detection use crude antigens obtained from partially purified $A$. marginale and thus lack the required sensitivity or specificity for a reliable diagnosis. Specific and sensitive polymerase chain reaction was developed to detect $A$. marginale DNA from animal blood and ticks which is thought to be more practical technique for diagnosis of the disease in domestic animals [4]. There were only a few previous reports on the prevalence of bovine anaplasmosis in Punjab [4] and as the propensity of tick population is higher in hilly and undulating areas, the present study targeted those areas of Punjab in particular. To the best of our knowledge, there is no previous report on the seroprevalence of $A$. marginale from Punjab. Hence, in the present investigation, bovine anaplasmosis due to $A$. marginale was comparatively evaluated by microscopy, PCR, and indirect ELISA in Submountain and Undulating Zone of Punjab to assess the level of exposure of animals in these two highly conducive zones of Punjab in relation to the risk factors associated with disease occurrence.

\section{Materials and Methods}

2.1. Study Area and Sampling. Punjab state is divided into five major agroclimatic zones according to their soil type, agricultural development, and precipitation and temperature indices. A representative bovine samples collection was done from March 2011 to September 2013 from the major agroclimatic zones of Punjab. Samples from hilly and undulating regions of Punjab, namely, Submountain and Undulating Zone, were selected for the study to screen the bovines with tick infestation, fever, jaundice, or anaemia for anaplasmosis. Blood $(\sim 3 \mathrm{~mL})$ was drawn into anticoagulant-coated and anticoagulant-free vacutainers. Samples were processed for thin smears, nucleic acid, and sera. Data on the characteristic of sampled animals (species, age, and health status) and farms (management and location) was obtained on predesigned questionnaire during sampling.

2.2. Sampling Frame. To study the status of molecular and serological prevalence of the disease, the expected prevalence to be $50 \%$ with confidence limits of $95 \%$ and a desired absolute precision of $5 \%$ to collect maximum number of samples was considered. The number of samples thus calculated was adjusted for finite population and correlated with 184 samples ( 74 cattle and 21 buffalo; 55 cattle calves and 34 buffalo calves) collected.

2.3. Microscopy. From the blood samples of all the selected animals, thin blood smears were made, air dried, fixed in methyl alcohol for $2 \mathrm{~min}$, and stained with working dilution of $10 \%$ Giemsa stain for $30 \mathrm{~min}$. The smears were then washed with tap water to remove extra stain, air dried, and examined under oil immersion for demonstration of $A$. marginale. The blood samples were further stored at $-20^{\circ} \mathrm{C}$ for DNA extraction.

2.4. Serological Study (iELISA). Indirect ELISA (targeting gene encoding $19 \mathrm{kD}$ protein) was carried out using
SVANOVIR A. marginale-Antibody test kit. In brief, field sample, positive and negative control sera were prediluted in 1:100 in 1x Phosphate Buffer Saline Tween (PBST). $100 \mu \mathrm{L}$ of prediluted tested plasma was added to the selected wells and the plate was sealed and incubated at $37^{\circ} \mathrm{C}$ for 30 minute. After $30 \mathrm{~min}$, the plate was washed four times with 1x PBST buffer. $100 \mu \mathrm{L}$ of horse reddish peroxidase conjugated with anti-bovine IgG monoclonal antibody diluted conjugate was added to all the wells. Plate was sealed and incubated at $37^{\circ} \mathrm{C}$ for $30 \mathrm{~min}$. Again the plate was washed four times with PBST buffer. $100 \mu \mathrm{L}$ of reconstituted ABTS substrate solution was added to all the wells. The sealed plate was incubated at $25^{\circ} \mathrm{C}$ for $30 \mathrm{~min}$ at room temperature. The reaction was concluded with stop solution containing $1 \%$ SDS. The results were read in a spectrophotometer (Tecan Nano Quant Infinite M200) at $405 \mathrm{~nm}$ filter within $15 \mathrm{~min}$ of stopping the reaction. A percent positivity (PP) of negative control and samples was calculated as follows:

$$
\mathrm{PP}=\frac{\text { Mean OD value negative control }}{\text { Mean OD value positive control }} \times 100 \text {. }
$$

For the interpretation of results, OD of 1.0-2.3 for positive control and $\mathrm{PP}<20$ for negative control were the criteria for test validity. Results of test samples were considered positive for $\mathrm{PP} \geq 40$ positive.

2.5. Polymerase Chain Reaction. For conducting the PCR assay, whole-genomic DNA was isolated from blood sample using HiPura Blood Genomic DNA Miniprep Purification Kit following the manufacturer's recommendations. Genomic DNA of A. marginale isolated from infected blood showing high parasitaemia was utilized as positive control. Genomic DNA was also isolated from the whole blood of infection-free day-old bovine calf and used as a negative control.

2.5.1. msp1 $\beta P C R$. DNA was extracted using HiPura Blood Genomic DNA Miniprep Purification Kit as per the protocol of the manufacturer. Amount of extracted DNA and its purity was measured at $\mathrm{OD}_{260}$ and ratio of $\mathrm{OD}_{260}$ to $\mathrm{OD}_{280}$, respectively. The BAP-2 and AL34S set of oligonucleotide primer was used to amplify mspl $\beta$ gene of $A$. marginale. The nucleotide sequence of the primer [5] is as follows:

\section{BAP-2: 5' GTA TGG CAC GTA GTC TTG GGA TCA $3^{\prime}$, \\ AL34S: 5' CAG CAG CAG CAA GAC CTT CA 3'.}

The $25 \mu \mathrm{L}$ PCR reaction mixture constituted $12.5 \mu \mathrm{L}$ of KAPA2G Fast Hot Start Ready Mix (2x containing KAPA2G Fast Hot Start DNA polymerase, KAPA2G Fast Hot Start PCR buffer, $0.2 \mathrm{mM}$ dNTP each, $1.5 \mathrm{mM} \mathrm{MgCl}_{2}$ ) with $1.5 \mu \mathrm{L} / 0.6 \mu \mathrm{M}$ of BAP-2/AL34S primers (10 pmol) suspended in nuclease-free water with $5 \mu \mathrm{L}$ DNA template in automated thermocycler (Eppendorf, mastercycler personal) with the following programme: initial denaturation at $95^{\circ} \mathrm{C}(5 \mathrm{~min})$, 30 cycles of denaturation at $95^{\circ} \mathrm{C}(1 \mathrm{~min})$, annealing at $60^{\circ} \mathrm{C}$ $(1 \mathrm{~min})$, and extension at $72^{\circ} \mathrm{C}(1.5 \mathrm{~min})$ with final extension 
TABLE 1: Incidence of A. marginale among different districts and animal groups under study as diagnosed by Blood Smear Examination (BSE), Enzyme Linked Immunosorbent Assay (ELISA), and Polymerase Chain Reaction (PCR).

\begin{tabular}{|c|c|c|c|c|c|c|c|c|c|}
\hline \multirow[b]{2}{*}{ Factors } & \multirow[b]{2}{*}{ Total } & \multicolumn{2}{|c|}{ BSE } & \multicolumn{2}{|c|}{ ELISA } & \multicolumn{2}{|c|}{ PCR } & \multicolumn{2}{|c|}{ Odd's ratio } \\
\hline & & $\begin{array}{l}\text { Positive } \\
(\%)\end{array}$ & $95 \% \mathrm{CI}$ & $\begin{array}{l}\text { Positive } \\
(\%)\end{array}$ & $95 \%$ CI & $\begin{array}{c}\text { Positive } \\
(\%)\end{array}$ & $95 \%$ CI & $\begin{array}{l}\text { (In terms } \\
\text { of PCR) }\end{array}$ & $95 \% \mathrm{CI}$ \\
\hline \multicolumn{10}{|l|}{ Zones } \\
\hline Submountain Zone & 109 & $16(14.67)$ & $8.95-20.41$ & 27 (24.77) & $17.78-31.75$ & $54(49.54)$ & $41.44-57.63$ & 2.086 & $1.81-4.042$ \\
\hline Undulation Zone & 75 & $9(12.0)$ & $5.65-18.34$ & $20(26.67)$ & $18.03-35.29$ & $24(32)$ & $22.89-41.10$ & 0.479 & $0.247-925$ \\
\hline \multicolumn{10}{|l|}{ Province } \\
\hline Gurdaspur & 41 & 8 (19.51) & $9.05-29.97$ & $13(31.71)$ & $19.42-43.98$ & $16(39.02)$ & $26.14-51.89$ & 0.836 & $0.387-1.797$ \\
\hline Hoshiarpur & 36 & $6(16.66)$ & $6.17-27.16$ & $6(16.67)$ & $6.17-27.16$ & $17(47.22)$ & $33.16-61.28$ & 1.276 & $0.712-2.819$ \\
\hline Pathankot & 32 & $2(6.25)$ & $-0.98-13.48$ & $8(25.0)$ & $12.06-37.93$ & $21(65.62)$ & $51.43-79.81$ & 3.182 & $1.341-7.656$ \\
\hline Rupnagar & 35 & $3(8.57)$ & $0.57-16.56$ & $9(25.71)$ & $13.23-38.19$ & $13(37.14)$ & $23.34-50.94$ & 0.764 & $0.334-1.732$ \\
\hline Nawanshahr & 40 & $6(15.0)$ & $5.45-24.54$ & $11(27.5)$ & $15.56-39.43$ & $11(27.50)$ & $15.56-39.43$ & 0.436 & $0.188-0.995$ \\
\hline \multicolumn{10}{|l|}{ Species } \\
\hline Cattle & 129 & $22(17.05)$ & $11.46-22.65$ & $37(28.68)$ & $21.95-35.41$ & $64(49.61)$ & $42.17-57.05$ & 2.884 & $1.364-6.165$ \\
\hline Buffalo & 55 & $3(5.45)$ & $0.27-10.63$ & $10(18.18)$ & $9.39-26.97$ & $14(25.45)$ & $15.53-35.38$ & 0.347 & $0.162-0.733$ \\
\hline \multicolumn{10}{|l|}{ Age } \\
\hline >1 year & 95 & 15 (15.78) & $9.46-22.11$ & $26(27.36)$ & 19.64-35.09 & 49 (51.57) & $42.92-60.24$ & 2.204 & $1.161-4.198$ \\
\hline$<1$ year & 89 & $10(11.23)$ & $5.57-16.89$ & $21(23.59)$ & $15.98-31.20$ & $29(32.58)$ & $24.18-40.98$ & 0.454 & $0.288-0.862$ \\
\hline
\end{tabular}

at $72^{\circ} \mathrm{C}$ for $5 \mathrm{~min}$. The amplified PCR products were separated by electrophoresis on $1 \%$ agarose gel and visualized under a UV Transilluminator for detection of $407 \mathrm{bp}$ amplified product.

2.5.2. Small Subunit (16S) rRNA nPCR. The conflicting samples positive by microscopy (being gold standard) and negative by mspl $\beta$ PCR were reconfirmed by $16 \mathrm{~S}$ rRNA nPCR. The P1 and P2 set of oligonucleotide primer was used in primary PCR cycle and P1 and P3 were used in secondary PCR cycle targeting $16 \mathrm{~S}$ rRNA gene of $A$. marginale. The nucleotide sequence of the primer [6] is as follows:

\section{P1: 5'-AGAGTTTGATCCTGGCTCAG-3', P2: 5'-AGCACTCATCGTTTACAGCG-3', P3: $5^{\prime}$-GTTAAGCCCTGGTATTTCAC-3'.}

The $25 \mu \mathrm{L}$ PCR reaction mixture constituted $12.5 \mu \mathrm{L}$ of KAPA2G Fast Hot Start Ready Mix (2x containing KAPA2G Fast Hot Start DNA polymerase, KAPA 2G Fast Hot Start PCR buffer, $0.2 \mathrm{mM}$ dNTP each, $1.5 \mathrm{mM} \mathrm{MgCl}_{2}$ ) with $1.5 \mu \mathrm{L} / 0.6$ $\mu \mathrm{M}$ of P1-P2 (or P1-P3 for nested) primers (10 pmol) suspended in nuclease-free water with $5 \mu \mathrm{L}$ of DNA template (or $2 \mu \mathrm{L}$ of Primary PCR product) in automated thermocycler (Eppendorf, mastercycler personal) with the following programme: initial denaturation at $95^{\circ} \mathrm{C}(5 \mathrm{~min}), 30$ cycles of denaturation at $95^{\circ} \mathrm{C}(1 \mathrm{~min})$, annealing at $57^{\circ} \mathrm{C}(1 \mathrm{~min})$, and extension at $72^{\circ} \mathrm{C}(1.5 \mathrm{~min})$ with final extension at $72^{\circ} \mathrm{C}$ ( $5 \mathrm{~min})$. The amplified PCR products were separated by electrophoresis on $1 \%$ agarose gel and visualized under a UV Transilluminator for detection of $564 \mathrm{bp}$ amplified product.
2.6. Analysis of Nucleotide Sequence. The amplicons were custom sequenced from Xcelris Genomics, Ahmedabad, India. The nucleotide sequences were subjected to BLASTn analysis [7] for determining the similarity with the sequences present in the nucleotide database. The nucleotide sequence alignment was studies by ClustalW software.

2.7. Statistical Analysis. Chi-square test was employed to relate the association of prevalence of infection with the different low lying areas and different groups of dairy animals under study on the basis of species and age. Win Episcope 2.0 software was applied to evaluate the agreement between the results revealed by different diagnostic tests used to detect A. marginale in this study. The risk factors were evaluated by calculation of Odd's ratio for all possible two way interactions. To identify goodness of fit, the observed versus predicted values (residual statistics) were derived by Win Episcope 2.0 software.

\section{Result and Discussion}

In the current study, the occurrence of $A$. marginale organisms, anti- $A$. marginale IgG antibodies, and $A$. marginale DNA in the blood of bovines from Submountain Zone and Undulating Zone of Punjab was investigated by microscopy, indirect ELISA, and mspl $\beta$ PCR, respectively. The study results depicted high frequency of this rickettsial organism among the ruminants in the region studied. PCR revealed incidence of $A$. marginale significantly highest in District Pathankot (65.62\%) (95\% CI $=51.43-79.81 \%)$ of Submountain Zone and lowest in Nawanshahr (27.50\%) 


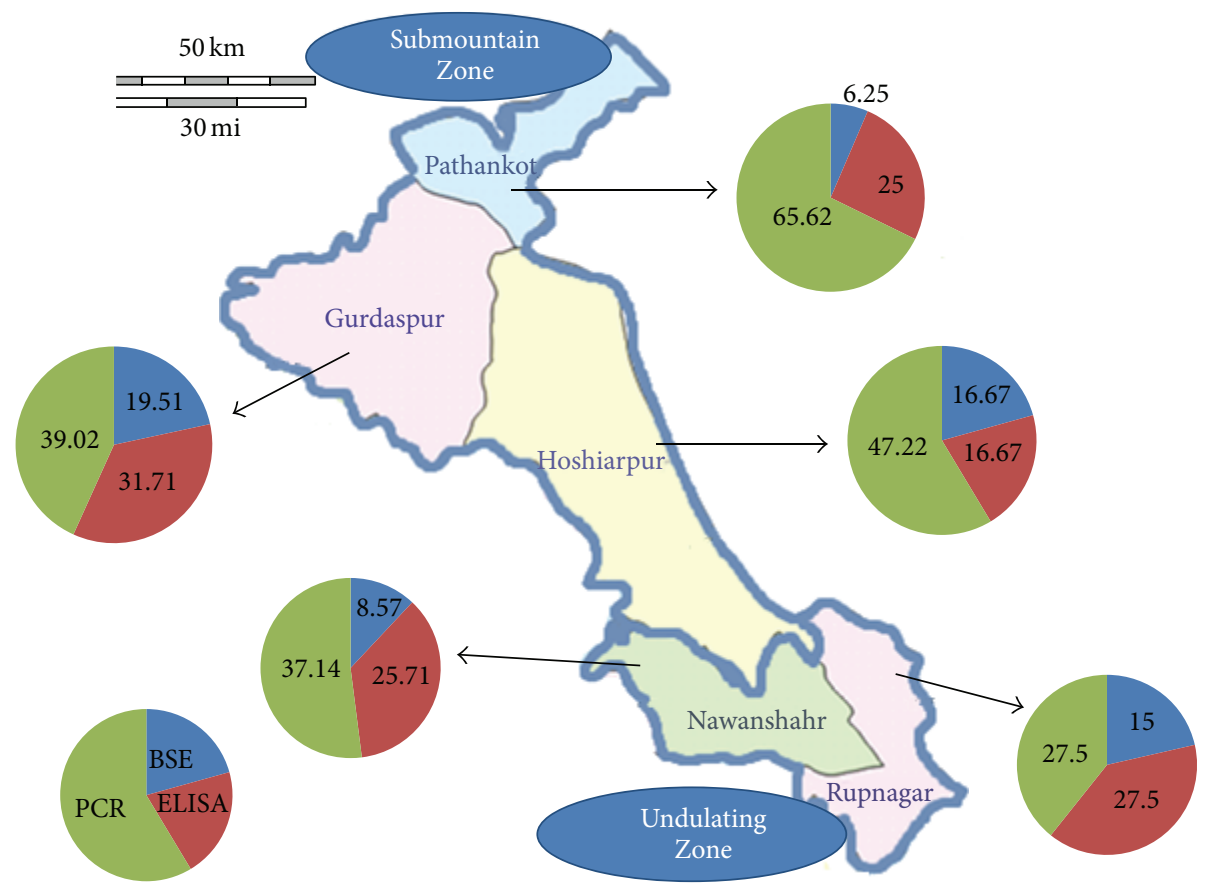

FIGURE 1: Map of Submountain and Undulatng Zone of Punjab indicating the incidence of A. marginale among different districts under study as diagnosed by Blood Smear Examination (BSE), Enzyme Linked Immunosorbent Assay (ELISA), and Polymerase Chain Reaction (PCR).

(95\% CI $=15.56-39.43 \%)$ of Undulating Zone (Table 1). The overall prevalence of $A$. marginale by $\mathrm{mspl} \beta$ PCR was $42.39 \%$ (95\% CI $=36.23-48.54 \%)$. The difference in the prevalence did not vary significantly among various districts under study as diagnosed by microscopy and ELISA. However, both tests showed highest incidence of anaplasmosis in district Gurdaspur of Submountain Zone (19.51\%, 31.71\%) (95\% CI $=9.05-29.97 \%$; 95\% CI $=19.42-43.98 \%$ ) (Table 1, Figure 1 ). All three districts of Submountain Zone are at higher risk indicating the impact of biotic and abiotic factors on the incidence of disease in relation to the disease incidence. Temperature and humidity are well known crucial factors for the development of major vector B. microplus, and the climate factor seems to be determinant for the distinct epidemiological conditions found in the semihumid region of Submountain Zone of Punjab. The total normal rainfall of the Kandi region varies from about 800 to $1500 \mathrm{~mm}$, about three-fourths of which is received during rainy season in a few rainy days (http://dolr.nic.in/dolr/downloads/spsp/SPSPPunjab.pdf). The parasitological and molecular prevalence of A. marginale revealed in this study is closed to a previous study conducted in Punjab [4]. The areas covered in the present study have comparatively higher prevalence of $B$. microplus [8] as an effect of favorable macroclimatic factors, thus posing higher threats of anaplasmosis in the area.

Between the two species under study, cattle were shown to have higher susceptibility/exposure to the disease as revealed by serological $(28.68 \%$; $95 \%$ CI $=21.95-35.41 \%)$ as well as molecular test (49.61\%; $95 \% \mathrm{CI}=11.23-35.82 \%$ ). The incidence of disease varied significantly among the four animal groups with the PCR, highest being in cattle adults $(58.11 \%)(95 \% \mathrm{CI}=48.41-67.80 \%)$ and lowest being

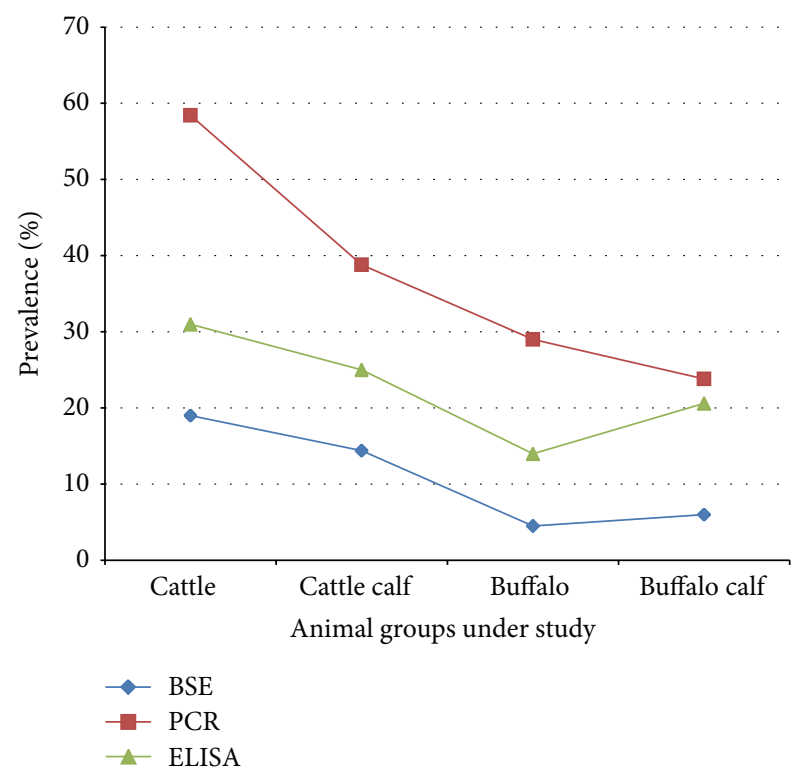

FIGURE 2: Incidence of $A$. marginale among different animal groups under study as diagnosed by Blood Smear Examination (BSE), Enzyme Linked Immunosorbent Assay (ELISA), and Polymerase Chain Reaction (PCR) revealing cattle as the most exposed group.

in buffalo calves (23.57\%) (95\% CI $=11.23-35.82 \%)$ (Table 1 , Figure 2). However, by microscopy and ELISA, there existed nonsignificant difference in the incidence of disease among the four animal groups. Cattle showed highest incidence of disease both by microscopy $(18.92 \%)(95 \% \mathrm{CI}=11.22-26.61 \%)$ 


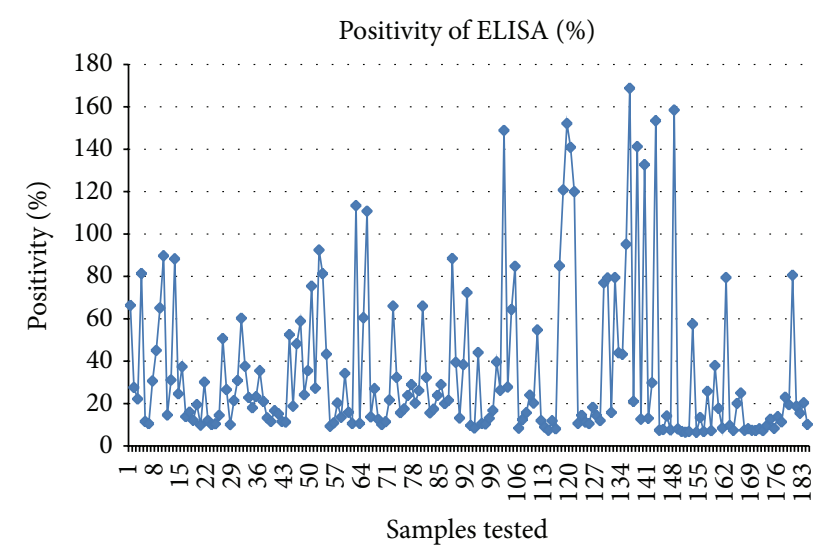

FIGURE 3: Percent positive plot of samples tested for anti- $A$. marginale antibody by indirect ELISA.

and ELISA (31.08\%) (95\% CI = 21.98-40.17\%); however, study explicates the role of buffalo as the potent carrier animal by not exhibiting any clinical symptoms but still harbouring the infection. Cattle displayed a higher incidence of disease than buffaloes by all the three diagnostic techniques. Cattle were found to be more prone to anaplasmosis infection as compared to their calves. Similar trend was seen among buffaloes as revealed by PCR; however, the detection based on microscopy and ELISA depicted higher incidence of the disease in buffalo calves (Figure 2). Significant difference in the seroprevalence of $A$. marginale among different groups [9] may be influenced by factors such as race, age, and physiological and immunological status. It is noteworthy to mention that parasite inoculation rate by other biological vectors [10-12] and other sources of mechanical transmission, namely, blood-sucking dipterans and fomites [13] may also affect the enzootic stability of any geographic area when $A$. marginale is prevalent. Additionally, the lowest seropositivity $(p<0.05)$ seen in cattle aged up to 12 months can be explained by immune protection due to colostral antibodies and/or low rate of inoculation of $A$. marginale by the vectors as the animals in this age group have much lower contact with the vectors compared to older ones [14].

The positivity by the three techniques markedly differed revealing percent positivity of $13.58 \%(n=25)(95 \%$ CI $=9.31-17.85 \%)$ by microscopy, $25.54 \%(n=47)(95 \% \mathrm{CI}$ $=20.11-30.97 \%)$ by ELISA, and $42.39 \%(n=78)(95 \% \mathrm{CI}$ $=36.23-48.54 \%$ ) by PCR (Table 2). Out of the 184 sample tested, 12 were positive and 77 were negative by all the three tests indicating $48.36 \%$ concordance between these tests (Table 2). Forty-nine samples were exceedingly positive by PCR indicating its higher sensitivity over microscopy and ELISA. Twenty samples negative by PCR could be detected positive by ELISA which indicated persistence of antibodies after clearance of infection, while 57 animals which were detected positive by PCR and negative by ELISA may indicate recent infection or may be carrying too low parasitemia to produce detectable antibodies. Out of the 47 samples positive by ELISA, the percent positive of serum antibody in animals positive by all the three tests $(n=12)$ was seen to be more

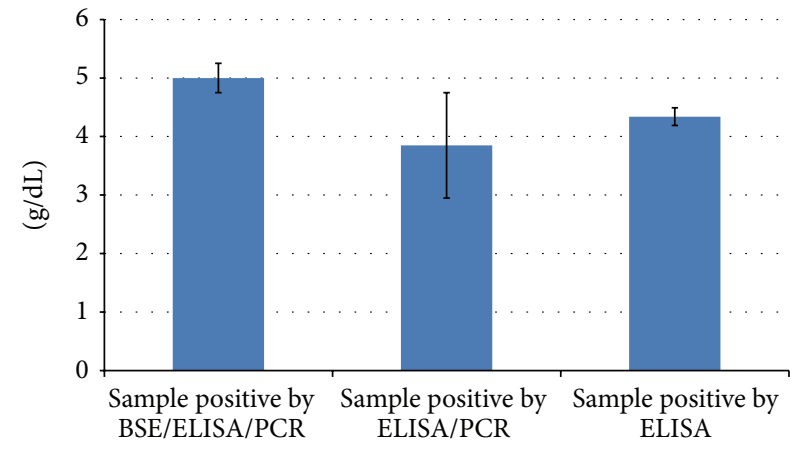

FIgURE 4: Serum globulin level in three groups tested positive by Blood Smear Examination (BSE), Enzyme Linked Immunosorbent Assay (ELISA), and Polymerase Chain Reaction (PCR).

than $100 \%$ (Figure 3), which was also corroborated with high globulin levels (Figure 4).

The assessment of risk factor with results of PCR technique as the reference indicates that cattle (Odd's ratio $=$ $2.884,95 \% \mathrm{CI}=1.364-6.165 \%)$, particularly those of age $>1$ years (Odd's ratio $=2.204,95 \% \mathrm{CI}=1.161-4.198 \%$ ) of district Pathankot (Odd's ratio $=3.182,95 \%$ CI $=1.341-7.656 \%$ ) of Submountain Zone (Odd's ratio $=2.086,95 \%$ CI $=1.81$ $4.042 \%)$ are at high risk of anaplasmosis caused by $A$. marginale.

Of twenty-five samples positive in slide examination, five cases were there which could not be detected positive by $\operatorname{mspl} \beta$ PCR, but 4 of those could show positive amplification by $16 \mathrm{~S}$ rRNA nPCR specific for Anaplasma spp. However, RFLP specific for A. marginale could not give conclusive results; that may be due to less concentration of PCR product or small difference in the size of PCR product and restriction digestion product. All four sequenced products revealed the presence of $A$. marginale specific restriction site (GTA $\downarrow$ TAC) in all the samples (Figure 5).

There may be a possibility that the mspl $\beta$ PCR showed negative results due to the polymorphism among geographic isolates of $A$. marginale [15] which was also seen in $A$. marginale Str. Florida (AF110809.1), Str. PR1 (EU281852.1), and Str. South Idaho (AF111196.1) (Figures 6(a) and 6(b)), the strain closely related to our local strain submitted under accession number KF696857 (previous study) (Figure 7).

Due to the limitation of degradation of mounted blood smear, one sample still negative by PCR could not be confirmed for $A$. marginale upon rechecking.

With PCR as the reference standard $(n=82)$, the sensitivity and specificity of microscopy $(n=25)$ were found to be $29 \%$ and $99 \%$ percent, while indirect ELISA targeting gene encoding $19 \mathrm{kD}$ protein $(n=47)$ showed sensitivity and specificity to be $32 \%$ and $79 \%$, respectively. There was a fair agreement between microscopy and PCR (Kappa = $0.304,95 \%$ CI $=0.202-0.326 \%)$ while a slight agreement between ELISA and PCR (Kappa $=0.093,95 \%$ CI $=-0.049$ $0.231 \%$ ), which may be because of antigen cross reactivity; thus, serological tests do not produce reliable results [16-18]. 
TABlE 2: Correlating between the findings of Blood Smear Examination (BSE), Enzyme Linked Immunosorbent Assay (ELISA), and Polymerase Chain Reaction (PCR).

\begin{tabular}{|c|c|c|c|c|c|}
\hline \multicolumn{6}{|c|}{ Polymerase chain reaction (PCR) } \\
\hline & & Positive & Negative & & \\
\hline \multirow{4}{*}{$\begin{array}{l}\text { Blood Smear } \\
\text { Examination (BSE) }\end{array}$} & Positive & $12^{\mathrm{abc}}$ & $2^{\mathrm{bc}}$ & Positive & \multirow{4}{*}{$\begin{array}{l}\text { Enzyme linked } \\
\text { immunosorbent } \\
\text { (ELISA) }\end{array}$} \\
\hline & Negative & $13^{\mathrm{ac}}$ & $20^{c}$ & Positive & \\
\hline & Positive & $8^{\mathrm{ab}}$ & $3^{\mathrm{b}}$ & Negative & \\
\hline & Negative & $49^{\mathrm{a}}$ & $77^{\mathrm{d}}$ & Negative & \\
\hline
\end{tabular}

Superscript "a" indicates samples positive by PCR, "b" indicates samples positive by blood smear examination, "c" indicates samples positive by ELISA, and "d" indicates samples negative by all three testes.

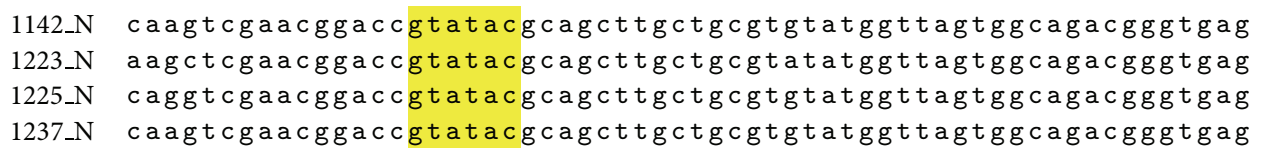

Figure 5: Multiple Sequence Alignment of the four samples positive by $16 \mathrm{~S}$ rRNA nPCR showing A. marginale specific restriction site (highlighted are the restriction sites GTATAC).

\begin{abstract}
AF110809.1 GCGTTCGGCAGCATATCCCATATCACCAACTCATTCGCCAAGTGGTAAGATGACAG 1692 AF110810.1 GCGTTCGGCAGCATATCCCATATCACCAACGTCATTCGCCAACTGGTAAGATCACAG 1692 EU281852.1 GCGTTCGGCAGCATATCCCATATCACCAACGTCATTCGCCAACTGGTAAGATCACAG 1668 AF348138.1 GCGT TCGGCAGCATATCCCATATCACCAACGTCATTCGCCAACTGGTAAGAGCACAG 1707 AF112479.1 GCGTTCGGCAGCATATCCCATATCACCAACGTCATTCGCCAACTGGTAAGATGACAG 1668 KF696857.1 - - - - - - ATGGCACGTAGTCTTGGGATCATGAGCAAAGTGGTAAGATGCACAG 48 AF221693.1 GTGATGT TCCGTATGGCACGTAGTCTTGGGATCATGAGCAAGCTAGTATAGAGGAAA 1761 AF110808.1 GTGATGT TCCGTATGGCACGTAGTCTTGGGATCATGAGCAAGCTAGTATAGAGGAAC 1770 AY841153.1 GTGATGTTCCGTATGGCACGTAGTCTTGGGATCATGAGCAAACTAGTATAGAGGAAC 1806 AF111195.1 GTGATGTTCCGTATGGCACGTAGTCTTGGGATCATGAGCAAGCTGGTATAGAGCAAC 1764 AF111197.1 GTGATGT TCCGTATGGCACGTAGTCTTGGGATCATGAGCAAGCTGGTATAGAGGAAC 1764 AF348137.1 GTGATGTTCCGTATGGCACGTAGTCTTGGGATCATGAGCAAACTAGTATAGAGGCAAC 1821 AF112480.1 GTGATGTCCGTATGGCACGTAGTCTTGGGATCATGAGCAAGCTAGTATAGAGGAAC 1752 M59845.1 GTGATGTTCCGTATGGCACGTAGTCTTGGGATCATGAGCAAGCTAGTATAGAGGAAC 2209 AF111196.1 GCGTTCGGCAGCATATCCCATATCACCAACGTCATTCGCCAAGTGGTAAGATGCACAG 1710 AF221692.1 GCGTTCGGCAGCATATCCCATATCACCAACGTATTCGCCAACTGGTAAGTGCACAG 3465 AF221691.1 _._._.
\end{abstract}

(a)

AF110809.1 GAAGG T C T T G C TA C T GCT T TAAA T GAGGCT T C T GCAGAAGGGAAAATACAAT C C C T CAA T AF110810.1 GAAGGTCT TGCTGCTGCTG TAAATGAGGCT T C TGCAGATGGGAAGATGCAGTCCCTCAAT EU281852.1 GAAGGTCT TGCTACTGCTGTAAATGAGGCT T C TACAGATGGGAAGATACAATCCCTCAAT AF348138.1 GAAGGTCT TGCTACTGCTGTAAATGAGGCT TCTGTAGATGGGAAAATACAATCCCCCAAT AF112479.1 GAAGG T C T T GCT T C T GCTGTAAATGAGGCT T C T GCAGATGGGAAGA TACAGT C C C T CAAT KF696857.1 AF221693.1 AF110808.1 AY841153.1 AF111195.1 AF111197.1 AF348137.1 AF112480.1 M59845.1 AF111196.1 AF221692.1 AF221691.1 GAAGGTCT T GCTGCTGCT G TAAATGAGGCT T C T GCAGATGGGAAGATGCAAT T C C T CAAA GA A G G T C T T G C T A C T G C T G T A A A T A G G C T T C T G CAGAAGGGAAAA T A CAA T C C C T CAA T CAAGGTCTTGCTACTGCTGTAAATGAGGCTTCTGCAGATGAGAAGATACAGTCCCTTAAT GAAGGTCTTGCTACT GCT T TAAATGAGGCT T C TGCAGAAGGGAAA TACAATCCCTCAAT

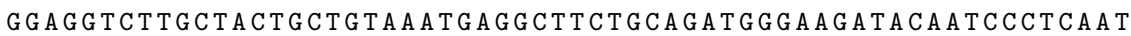
CAAGGT C T T GCTACTGCT G TAAATGAGGCT T C T GCAGATGGGAAGATA CAG T C C C T CAAT GAAGGTCTTGCTACTGCTGTAAATGAGGCTTCTGCAGATGGGAAGATACAGTCCCTCAAT GAAGGTCT TGCTGCTGCTGTAAATGAGGCT T C T GCAGATGGGAAGATGCAGTCCCTCAAT GAAGGTCTTGCTACTGCTGTAAATGAGGCTTCTGCAGAAGGGAAAATACAATCCTTCAAT GAAGG T C T T GCTACTGCT G TAAATGAGGCT T C T GCAGAAGGGAAAATACAATCCCTCAAT

(b)

Figure 6: (a) Multiple sequence alignment of various geographic isolates of $A$. marginale showing variations at forward primer binding site of $\mathrm{mspl} \beta$ used in the present study. (b) Multiple sequence alignment of various geographic isolates of $A$. marginale showing variations at reverse primer binding site of $\operatorname{msp} 1 \beta$ used in the present study. 


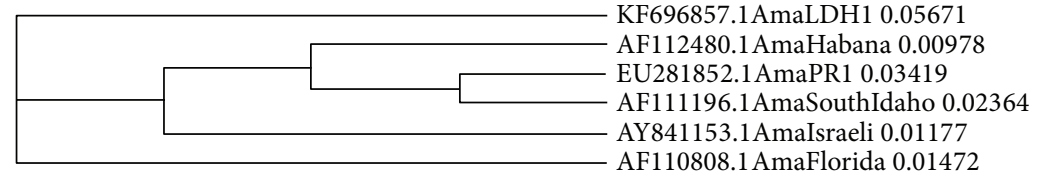

FIgURE 7: Phylogenetic relationship of local strain (KF696857.1) for the partial msplb coding sequences of different strains of Anaplasma marginale.

The results of the current study demonstrate the possible variation in the mspl $\beta$ gene among the isolates of Punjab and also depict great transmission potential of $A$. marginale in the area targeted. MSPs as important tools for recombinant protein, monospecific and monoclonal antibodies, isolate variability, and potential value in diagnostic assays and vaccines [15], thus the present study may provide future directions for control measures. These data may provide valuable input to managers of livestock and can help understanding the status of herds as well as for planning future interventions strategies. Further the findings of molecular prevalence of $A$. marginale indicated that cattle, particularly those of age $>1$ year of district Pathankot of Submountain Zone, are at high risk of anaplasmosis caused by $A$. marginale, while the overall incidence of the disease was associated with the geographic location of the areas under study.

\section{Conflict of Interests}

All the authors declare no financial or personal conflict of interests with the faculty or any other institute.

\section{Acknowledgment}

Thanks are due to the Director of Research, Guru Angad Dev Veterinary and Animal Sciences University, for providing funds under RKVY project entitled Employment of ImmuneMolecular Diagnostic Tools for Haemoprotozoans in Livestock of Punjab".

\section{References}

[1] M. Ristic, "Anaplasmosis," in Infectious Blood Diseases of Man and Animals, D. Weinman and M. Ristic, Eds., pp. 473-542, Academic Press, New York, NY, USA, 1968.

[2] R. M. Lewis and C. A. Picut, Eds., Veterinary Clinical Immunology: From Classroom to Clinics, Lea \& Febiger, Philadelphia, $\mathrm{Pa}$, USA, 1989.

[3] L. O. Mott, "The nature of anaplasmosis", in Proceedings of the 3rd National Anaplasmosis Conference, pp. 1-9, Manhattan, Kan, USA, 1957.

[4] A. Sharma, L. D. Singla, P. Kaur, M. S. Bal, B. K. Batth, and P. D. Juyal, "Prevalence and haemato-biochemical profile of Anaplasma marginale infection in dairy animals of Punjab (India)," Asian Pacific Journal of Tropical Medicine, vol. 6, no. 2, pp. 139144, 2013.

[5] U. G. Munderloh, C. M. Tate, M. J. Lynch, E. W. Howerth, T. J. Kurtti, and W. R. Davidson, "Isolation of an Anaplasma sp. organism from white-tailed deer by tick cell culture," Journal of Clinical Microbiology, vol. 41, no. 9, pp. 4328-4335, 2003.

[6] V. Noaman and P. Shayan, "A new PCR-RFLP method for detection of Anaplasma marginale based on 16S rRNA," Veterinary Research Communications, vol. 34, no. 1, pp. 43-50, 2010.

[7] S. F. Altschul, W. Gish, W. Miller, E. W. Myers, and D. J. Lipman, "Basic local alignment search tool," Journal of Molecular Biology, vol. 215, no. 3, pp. 403-410, 1990.

[8] A. P. Singh, L. D. Singla, and A. Singh, "A study on the effects of macroclimatic factors on the seasonal population dynamics of Boophilus micropus (Canes, 1888) infesting the cross-bred cattle of Ludhiana district," International Journal of Animal Science, vol. 15, no. 1, pp. 29-31, 2000.

[9] R. E. Bock, A. J. de Vos, T. G. Kingston, and D. J. McLellan, "Effect of breed of cattle on innate resistance to infection with Babesia bovis, B bigemina and Anaplasma marginale," Australian Veterinary Journal, vol. 75, no. 5, pp. 337-340, 1997.

[10] K. Dreyer, L. J. Fourie, and D. J. Kok, "Epidemiology of tickborne diseases of cattle in Botshabelo and Thaba Nchu in the Free State Province," Onderstepoort Journal of Veterinary Research, vol. 65, no. 4, pp. 285-289, 1998.

[11] M. S. Mtshali, D. T. De Waal, and P. A. Mbati, "A sero-epidemiological survey of blood parasites in cattle in the northeastern Free State, South Africa," Onderstepoort Journal of Veterinary Research, vol. 71, no. 1, pp. 67-75, 2004.

[12] T. P. Ndou, B. M. Dzoma, and L. E. Motsei, “The seroprevalence and endemic stability of anaplasmosis in cattle around Mafikeng in the North West Province, South Africa," Veterinary Research, vol. 3, no. 1, pp. 1-3, 2010.

[13] G. A. Scoles, A. B. Broce, T. J. Lysyk, and G. H. Palmer, "Relative efficiency of biological transmission of Anaplasma marginale (Rickettsiales: Anaplasmataceae) by Dermacentor andersoni (Acari: Ixodidae) compared with mechanical transmission by Stomoxys calcitrans (Diptera: Muscidae)," Journal of Medical Entomology, vol. 42, no. 4, pp. 668-675, 2005.

[14] N. N. Jonsson, R. E. Bock, and W. K. Jorgensen, "Productivity and health effects of anaplasmosis and babesiosis on Bos indicus cattle and their crosses, and the effects of differing intensity of tick control in Australia," Veterinary Parasitology, vol. 155, no. 1-2, pp. 1-9, 2008.

[15] K. M. Kocan, J. De la Fuente, A. A. Guglielmone, and R. D. Meléndez, "Antigens and alternatives for control of Anaplasma marginale infection in cattle," Clinical Microbiology Reviews, vol. 16, no. 4, pp. 698-712, 2003.

[16] D. S. Bradway, S. T. de Echaide, D. P. Knowles, S. G. Hennager, and T. F. McElwain, "Sensitivity and specificity of the complement fixation test for detection of cattle persistently infected with Anaplasma marginale," Journal of Veterinary Diagnostic Investigation, vol. 13, no. 1, pp. 79-81, 2001. 
[17] J. de la Fuente, A. Torina, S. Caracappa et al., "Serologic and molecular characterization of Anaplasma species infection in farm animals and ticks from Sicily," Veterinary Parasitology, vol. 133, no. 4, pp. 357-362, 2005.

[18] U. M. Dreher, R. Hofmann-Lehmann, M. L. Meli et al., "Seroprevalence of anaplasmosis among cattle in Switzerland in 1998 and 2003: no evidence of an emerging disease," Veterinary Microbiology, vol. 107, no. 1-2, pp. 71-79, 2005. 

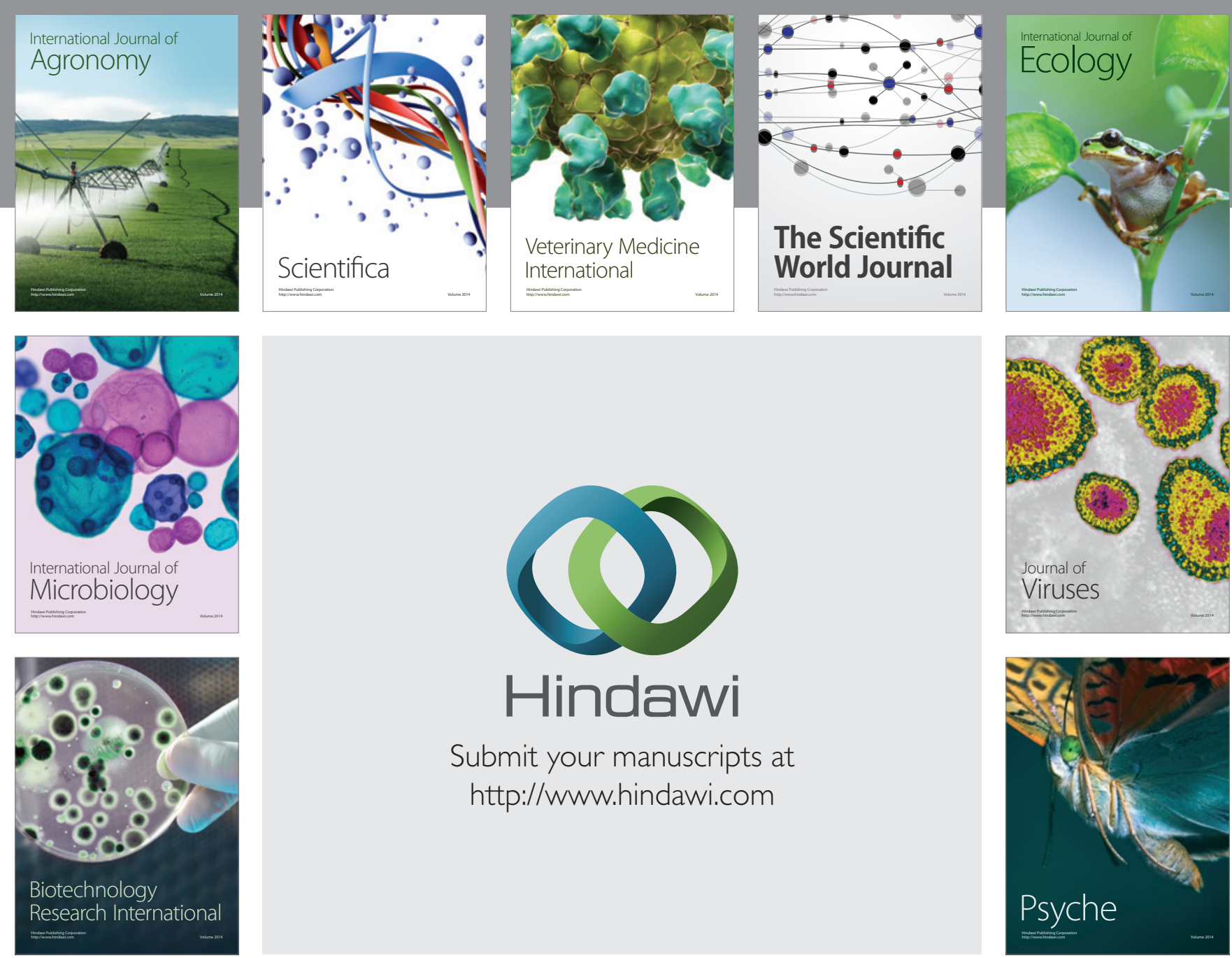

Submit your manuscripts at http://www.hindawi.com
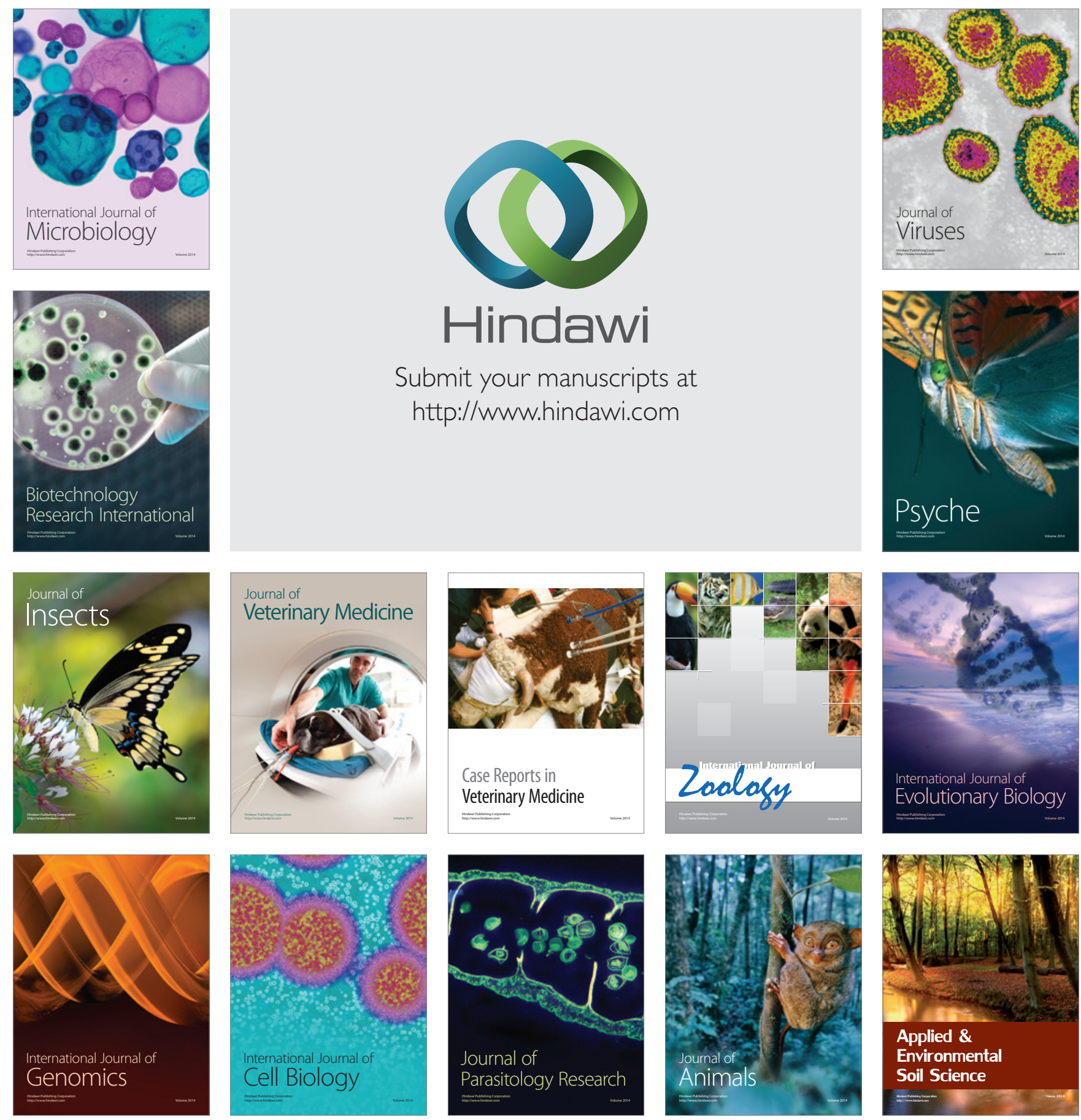